\title{
A Biomimetic-Computational Approach to Optimizing the Quantum Efficiency of Photovoltaics
}

\author{
Lisa M. Perez ${ }^{1}$ and Andreas Holzenburg ${ }^{2}$ \\ ${ }^{1}$ Laboratory for Molecular Simulation, Texas A\&M University, College Station, TX 77843- \\ 3012, U.S.A.; \\ ${ }^{2}$ Microscopy \& Imaging Center, Department of Biology, Department of Biochemistry and \\ Biophysics, Texas A\&M University, College Station, TX 77843-2257, U.S.A.
}

The most advanced low-cost organic photovoltaic cells have a quantum efficiency of $\sim 10 \%$. This is in stark contrast to plant/bacterial light-harvesting systems which offer quantum efficiencies close to unity. Of particular interest is the highly effective quantum coherence-enabled energy transfer. Noting that quantum coherence is promoted by charged residues and local dielectrics, classical atomistic simulations and Time-Dependent Density Functional Theory (TD-DFT) [1] can be used to identify charge/dielectric patterns and electronic coupling at exactly defined energy transfer interfaces incorporating structural information obtained on photosynthetic protein-pigment complexes. To this end, the project focuses on the first protein-pigment-redox carrier complex of the linear electron transport phosphorylation chain termed photosystem II [PSII] [2]. PSII contains more than 10 major polypeptides in addition to hundreds of pigment molecules amounting to a molecular mass in excess of 1 Mio Dalton. Owing to the complexity and fragility of PSII, this project bases the overall architecture of PSII on in situ EM data providing structural clues about the entire, unperturbed PSII complex [3, 4]. Albeit not to high resolution when compared to X-ray crystallography and NMR spectroscopy, the EM tomographic results and projection maps provide an accurate delineation of the native complex suitable for fitting high-resolution X-ray data of PSII subcomplexes (Fig. 1) [5-8] towards an atomistic model of the entire PSII complex. This must also include the light-harvesting antennae, i.e. the light-harvesting chlorophyll (Chl) a/b protein complex [LHCII]. With respect to LHCII one should take into account positioning LHCII next to PSII as well as in a separate, complementary membrane thus permitting to test for both, horizontal (intramembrane) and vertical (intermembrane) energy transfer, respectively. The presence of LHCII in a membrane different from PSII is supported by strong biochemical evidence and tomographic data [3], and it has also been noted that the organization of LHCII may change in response to environmental conditions [9-11].

Theoretical investigations thus far have focused on UV-Vis spectra calculations for single Chla, Chlb, the strong coupling special Chla/Chla pair (D1/D2, the reaction center) and intermediate coupling Chlb/Chlb pairs in LHCII. As expected, the TD-DFT $\omega$ B97X-D [12] calculated spectra showed strong communication (strong red-shift) between the Chla/Chla pair that has a short Mg$\mathrm{Mg}$ distance (8 $\AA$ ) and varying degrees of weaker communication (little to no red-shift) for Chlb/Chlb pairs in the LHCII displaying Mg-Mg distances above $9 \AA$.

In order to move quantum-structured photovoltaics into the mainstream, it is important to identify dielectric patterns and changes therein at the quantum scale. With this strategy and the interdisciplinary combination of complementary fields of research depicted in Fig. 2, bandgap engineering of nanomaterials aimed at the replication of biosystems that have been optimized for millions of years together with the establishment of quantum coherence principles could constitute a perfect synergy towards the optimization of achievable target module efficiencies. 
References:

[1] Bauernschmitt, R., and Ahlrichs, R.: Chem. Phys. Lett. 256 (1996) p. 454.

[2] Wydrzynski, T.; Satoh, Kimiyuki (Eds.) (2005) Photosystem II. Advances in Photosynthesis and Respiration, Vol. 22, Springer, 786 pp.

[3] Ford R.C., Stoylova, S.S., and Holzenburg A.: Eur. J. Biochem. 269 (2002) p. 326.

[4] Stoylova, S.S., Ford, and R.C., Holzenburg, A.: Ultramicroscopy 77 (1999) p. 113.

[5] Umena, Y. et al.: Nature 473 (2011) p. 55.

[6] Loll, B. et al.: Nature 438 (2005) p. 1040.

[7] Liu, Z. et al.: Nature 428 (2004) p. 287.

[8] Pan, X.W. et al.: Nat. Struct. Mol. Biol. 18 (2011) p. 309.

[9] Ford, R.C., and Holzenburg, A.: Cryst. Res. Technol. 49 (2014) p. 637.

[10] Simidjiev, I., et al.: Proc. Natl. Acad. Sci. U.S.A. 97 (2000) p. 1473.

[11] Garab, G., et al.: Biochemistry 41 (2002) p. 15121.

[12] Chai, J.D., and Head-Gordon, M.: J. Chem. Phys. 128 (2008) 084106.

[13] The authors would like to acknowledge support by the Supercomputing Facility and the Division of Research (Texas A\&M University).

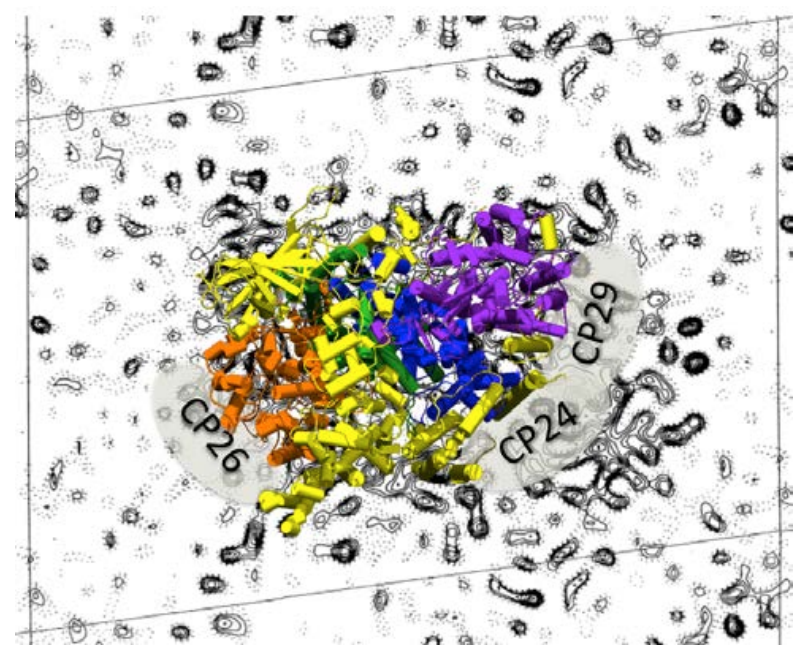

Figure 1. Synergy between X-ray and in situ electron crystallography. Conceptualizing the atomistic model of PSII. Subunit CP24, 26, and 29 positions are marked. One unit cell is shown with $\mathrm{a} \times \mathrm{b}=15.5 \times 23.1 \mathrm{~nm}$ and $\gamma=97.3^{\circ}$ [3].

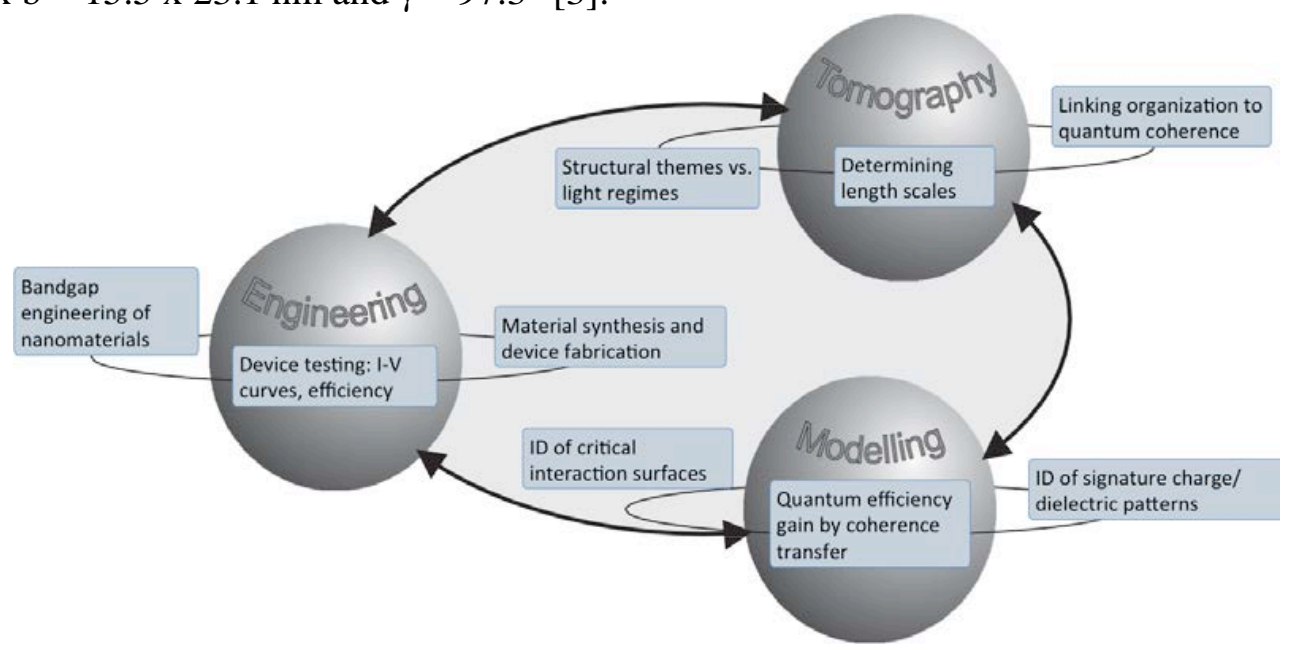

Figure 2. Interdisciplinary strategy critically involving complementary expertise. 